\title{
Mathematical and Conceptual Integration for Physics Quandaries
}

\author{
Mark V. Prizmic \\ Department of Mathematics \\ Irvine Valley College \\ Irvine, CA, U.S.A
}

\begin{abstract}
The intentions of this paper are to provide the reader with not only knowledge on general physics principles and theories, but with the tools necessary to compute and/or derive situations revolving around Ballistic Pendulums, Hooke's Law, and Buoyancy conundrums. Please note: For accuracy, significant figures may or may not be taken into account.
\end{abstract}

\section{Keywords}

Ballistic Pendulum, Hooke's Law, Buoyancy, theories, computations, algebra, trigonometry, proofs, variables, physics

\section{BALLISTIC PENDULUM THEORY}

A projectile launcher is capable of launching a steel ball consecutively while holding a constant initial velocity. The steel ball then has the possibility of colliding with the ballistic pendulum in a completely inelastic collision. At this point, however, only linear momentum is conserved; the summation of the system's mechanical energy is not conserved. When the collision is finished, the summation of the system's mechanical energy then becomes conserved.

$$
\begin{aligned}
& \text { 1.1 Ballistic Pendulum Variables } \\
& \mathrm{h}=\text { Height } \\
& \mathrm{R}=\text { Horizontal displacement } \\
& \mathrm{g}=\text { Gravity } \\
& \mathrm{m}=\text { Ball's mass } \\
& \mathrm{m}+\mathrm{M}=\text { Combined mass } \\
& R_{C M}=\text { Distance } \\
& v_{f}=\text { Final velocity magnitude after impact } \\
& v_{i}=\text { Initial velocity magnitude }
\end{aligned}
$$

\subsection{Critical Computations (3) to}

\section{Extrapolate Ballistic Pendulum Data}

1) Formula to compute final velocity magnitude after $\operatorname{impact}(\mathrm{m} / \mathrm{s}): V_{f}=\sqrt{2 g R_{C M}(1-\cos \theta)}$

Computing final velocity magnitude after impact example [medium of no mass]:

$\mathrm{V}_{\mathrm{f}}=\sqrt{2 \mathrm{gR}_{\mathrm{CM}}(1-\cos \theta)} ; \mathrm{g}=9.8 \mathrm{~m} / \mathrm{s}^{\wedge} 2, R_{C M}=.262 \mathrm{~m}, \theta=$ 45.8 degrees

$\rightarrow V_{f}=\sqrt{2(9.8)(.262)(1-\cos (45.8))} \rightarrow V_{f}=$ $\sqrt{1.5551177} \rightarrow V_{f} \approx 1.25 \mathrm{~m} / \mathrm{s}$
2) Formula to compute initial velocity magnitudemomentum conservation $(\mathrm{m} / \mathrm{s})$ in kilograms:

$$
V_{i}=\frac{m+M}{m} \sqrt{2 g R_{C M}(1-\cos \theta)}
$$

Computing initial velocity magnitude-momentum conservation $(\mathrm{m} / \mathrm{s})$ in kilograms example [medium of no mass]:

$\mathrm{V}_{\mathrm{i}}=\frac{\mathrm{m}+\mathrm{M}}{\mathrm{m}} \sqrt{2 \mathrm{gR}_{\mathrm{CM}}(1-\cos \theta)} ; \mathrm{g}=9.8 \mathrm{~m} / \mathrm{s}^{\wedge} 2, R_{C M}=.262 \mathrm{~m}$, $\theta=45.8$ degrees

$\rightarrow \mathrm{V}_{\mathrm{i}}=\frac{206.9}{158.9} \sqrt{2(9.8)(.262)(1-\cos (45.8))} \rightarrow \mathrm{V}_{\mathrm{i}}=$ $\frac{206.9}{158.9}(12.47) \approx 1.63 \mathrm{~m} / \mathrm{s}$

3) Formula to compute initial velocity magnitudeprojectile motion $(\mathrm{m} / \mathrm{s}): V_{i}=R \sqrt{\frac{g}{2 h}}$

Computing initial velocity magnitude-projectile motion example [medium of no mass]:

$$
\begin{aligned}
& V_{i}=R \sqrt{\frac{g}{2 h}} ; \mathrm{g}=9.8 \mathrm{~m} / \mathrm{s}^{\wedge} 2, \mathrm{~h}=.831 \mathrm{~m}, 1.344 \mathrm{~m} \\
& \rightarrow V_{i}=1.344 \sqrt{\frac{9.81}{2(.831)}} \approx 3.27 \mathrm{~m} / \mathrm{s}
\end{aligned}
$$

\section{HOOKE'S LAW THEORY}

Theory: The deformation a multitude of elastic substances undergo due to an external force acting on them is directly proportional to a restoring force that resists any further deformation. This relationship is known as Hooke's Law. When the motion of an object is repeated in regular time intervals, it is, defacto, undergoing periodic motion. Now, when oscillation occurs on a hanging mass, the motion is classified as simple harmonic motion. Do note that the wavelength will be defined as the distance between two adjacent crests/troughs.

$$
\begin{aligned}
& \text { 2.1 Hooke's Law Variables } \\
& F_{s}=\text { Hooke's Law } \\
& \Delta r=\text { Linear deformation } \\
& \mathrm{k}=\text { Stiffness of string } \\
& \mathrm{t}=\text { Instantaneous time } \\
& \mathrm{T}=\text { Period of oscillation } \\
& \mathrm{A}=\text { Amplitude displacement of hanging mass } \\
& \mathrm{m}=\text { Mass }
\end{aligned}
$$


$\mathrm{m}^{\prime}=$ Effective mass of spring when no hanging mass is attached to it

\subsection{Critical Computations (3) to extrapolate Spring Motion Data and \\ Hooke's Law Data}

1) Formula to compute Hooke's Law: $F_{S}=k \Delta r \mathrm{U}$ $F_{s}=k\left(r_{f}-r_{i}\right)$

Computing Hooke's Law example:

$F_{S}=k\left(r_{f}-r_{i}\right) ; \mathrm{k}=1.02, r_{f}=3.5, r_{i}=2.2$

$\rightarrow F_{S}=1.02(3.5-2.2) \rightarrow F_{S}=1.02(1.3)=1.327 \mathrm{~N}$

2) Formula to compute motion of hanging mass: $r=\operatorname{Acos}\left(\frac{2 \pi t}{T}\right)$

Computing motion of hanging mass example:

Prove "r," will equal 1 if $\mathrm{A}=2, \mathrm{t}=1$, and $\mathrm{T}=6$.

$r=\operatorname{Acos}\left(\frac{2 \pi t}{T}\right) ; \mathrm{A}=2, \mathrm{t}=1, \mathrm{~T}=6$

$\rightarrow r=2 \cos \left(\frac{(2 \pi) \times(1)}{6}\right) \rightarrow r=2 \cos \left(\frac{\pi}{3}\right)$

$\because \frac{\pi}{3} \in$ QuadI $\rightarrow \theta=+\frac{1}{2}$

$\rightarrow r=2\left(\frac{1}{2}\right)=1 \therefore r=1 \mathrm{~N} / \mathrm{m}$

Q.E.D

3) Formula to compute the period of oscillation:

$$
T=2 \pi \sqrt{\frac{m+m \prime}{k}}
$$

Computing period of oscillation example:

$T=2 \pi \sqrt{\frac{m+m^{\prime}}{k}} ; m=35.7, m^{\prime}=45, \mathrm{k}=1.05$

$\rightarrow T=2 \pi \sqrt{\frac{35.7+45}{1.05}} \approx 55.08 \mathrm{~Hz}$

\section{BUOYANCY THEORY}

Theory: An object immersed in a liquid will experience a positive, upward buoyant force which may cause it to float. In a liquid, the pressure on the lower surface of an object will always be stronger than on the upper surface such that there's a difference in pressure between the two surfaces. This produces the buoyant force which, by definition, is the upward-oriented force acting on the object. Archimedes' principle describes the buoyant force such that when an object is immersed into a liquid it's buoyed up by a force equal to the weight of liquid it displaces. That being said, Archimedes' principle is applicable to an object which is partially or completely submerged in a liquid.

\subsection{Buoyancy Variables}

$\mathrm{B}=$ Buoyant force

$\mathrm{P}=$ Pressure-depth relationship

$\rho_{L}=$ Mass density of the liquid

$g=$ Magnitude of the acceleration due to gravity $\mathrm{d}=$ Depth below surface of liquid

$V_{L}=$ Volume of liquid displaced by object's presense

$T_{\text {in }}=$ Magnitude of the tension in the string when the object is immersed in the liquid

$T_{\text {out }}=$ Magnitude of the tension in the string when the object is outside of the liquid

$\mathrm{m}=$ Mass

\subsection{Critical Computations (3) to Extrapolate Buoyancy Data}

1) Formula to compute buoyant force (magnitude): $B=m_{L} g \cup B=\left(\rho_{L} V_{L}\right) g$

Computing buoyant force example:

$B=\left(\rho_{L} V_{L}\right) g ; \rho_{L}=1.00, V_{L}=3.5, \mathrm{~g}=9.81$

$\rightarrow B=(1 \times 3.5) 9.81 \rightarrow B \approx 34.34 N$

2) Formula to compute pressure-depth relationship: $P=P_{L} g d$

Computing pressure-depth relationship example:

$P=\rho_{L} g d ; \rho_{L}=2.50, \mathrm{~g}=9.81, \mathrm{~d}=50$

$\rightarrow P=(2.50) \times(9.81) \times(50) \approx 1,226.3 \mathrm{~N} / \mathrm{m}^{\wedge} 2$

3) Deriving buoyant force:

Step 1: Derive $T_{\text {out }}$ using Newton's $2^{\text {nd }}$ Law

$\rightarrow \vec{F}_{\text {net }}=m \vec{a}$

$\rightarrow \vec{T}_{\text {out }}+\vec{W}=m \vec{a}$

$\rightarrow T_{\text {out }, y}+W_{y}=m a_{y}$

$\rightarrow T_{\text {out }}-W=0 \mathrm{~N}$

$\rightarrow T_{\text {out }}=W=m g$

Step 2: Derive $T_{\text {in }}$ using Newton's2nd Law

$\rightarrow \vec{F}_{\text {net }}=m \vec{a}$

$\rightarrow \vec{T}_{i n}+\vec{W}+\vec{B}=m \vec{a}$

$\rightarrow \vec{T}_{i n, y}+W_{y}+B_{y}=m a_{y}$

$\rightarrow T_{\text {in }}-W+B=0 N$

$\rightarrow T_{\text {in }}=W-B=m g-B$

Step 3: Use substitution to derive the buoyant force

$$
\begin{aligned}
& \rightarrow T_{\text {in }}=m g-B \\
& \rightarrow T_{\text {in }}=T_{\text {out }}-B \\
& \rightarrow \therefore \mathrm{B}=T_{\text {out }}-T_{\text {in }}
\end{aligned}
$$




\section{ACKNOWLEDGMENTS}

A big thanks to Nuwan Karunaratne for the advanced general education provided through several physics lectures and labs. A kind-hearted professor who never gave up on his students, and would always be more than glad to walk them through each problem. Without him none of this would have been possible.

\section{REFERENCES}

[1] Urone, Paul Peter., Roger Hinrichs, Kim Dirks, and Manjula Sharma. College Physics. Houston, TX: OpenStax College, Rice U, 2014. Print.

[2] Urone, Paul Peter., Roger Hinrichs, Kim Dirks, and Manjula Sharma. "Dynamics: Force and Newton's Laws of Motion." College Physics. Houston, TX: OpenStax College, Rice U, 2014. Print.

[3] Urone, Paul Peter., Roger Hinrichs, Kim Dirks, and Manjula Sharma. "Fluis Statics." College Physics. Houston, TX: OpenStax College, Rice U, 2014. Print.

[4] Urone, Paul Peter., Roger Hinrichs, Kim Dirks, and Manjula Sharma. "Introduction: The Nature of Science and Physics." College Physics. Houston, TX: OpenStax College, Rice U, 2014. Print.

[5] Urone, Paul Peter., Roger Hinrichs, Kim Dirks, and Manjula Sharma. "Oscillation Motion and Waves." College Physics. Houston, TX: OpenStax College, Rice U, 2014. Print. 